\title{
Risk of Mortality Associated with Non-selective Antimuscarinic medications in Older Adults with Dementia: a Retrospective Study
}

\author{
Nandita Kachru, PhD', Holly M. Holmes, MD, MS', Michael L. Johnson, PhD', \\ Hua Chen, $P h D^{7}$, and Rajender R. Aparasu, PhD, FAPhA' (D)
}

'Department of Pharmaceutical Health Outcomes and Policy, College of Pharmacy, University of Houston, Houston, TX, USA; ${ }^{2}$ Division of Geriatric and Palliative Medicine, McGovern Medical School at UTHealth, Houston, TX, USA.

\begin{abstract}
BACKGROUND: Selective antimuscarinics may offer a favorable safety profile over non-selective antimuscarinics for the management of overactive bladder (OAB) in patients with dementia.
\end{abstract}

OBJECTIVE: To test the hypothesis that non-selective antimuscarinics are associated with increased risk of mortality compared to selective antimuscarinics in older adults with dementia and OAB.

DESIGN: Propensity score-matched retrospective newuser cohort design among Medicare beneficiaries in community settings.

PATIENTS: Older adults with dementia and OAB with incident antimuscarinic use.

MAIN MEASURES: The primary exposure was antimuscarinic medications classified as non-selective (oxybutynin, tolterodine, trospium, fesoterodine) and selective (solifenacin, darifenacin) agents. All-cause mortality within 180 days of incident antimuscarinic use formed the outcome measure. New users of non-selective and selective antimuscarinics were matched on propensity scores using the Greedy $5 \rightarrow 1$ matching technique. Cox proportional-hazards model stratified on matched pairs was used to evaluate the risk of mortality associated with the use of non-selective versus selective antimuscarinics in the sample.

KEY RESULTS: The study identified 16,955 (77.6\%) nonselective antimuscarinic users and 4893 (22.4\%) selective antimuscarinic users. Propensity score matching yielded 4862 patients in each group. The unadjusted mortality rate at 180 days was $2.6 \%$ (126) for non-selective and $1.6 \%(78)$ for selective antimuscarinic users in the matched cohort ( $p$ value $<0.01$ ). The Cox model stratified on matched pairs found 50\% higher risk of 180-day mortality with non-selective antimuscarinics as compared to selective ones (hazard ratio (HR) 1.50; 95\% confidence interval (CI) 1.04-2.16). The study findings remained consistent across multiple sensitivity analyses.

CONCLUSIONS: Use of non-selective antimuscarinics was associated with a 50\% increase in mortality risk

Electronic supplementary material The online version of this article (https://doi.org/10.1007/s11606-020-05634-3) contains supplementary material, which is available to authorized users.

Received November 11, 2019

Revised November 23, 2019

Accepted January 3, 2020

Published online February 5, 2020 among older adults with dementia and OAB. Given the safety concerns regarding non-selective antimuscarinic agents, there is a significant need to optimize their use in the management of $\mathrm{OAB}$ for older patients with dementia.

KEY WORDS: dementia; overactive bladder; antimuscarinic; selective; non-selective; mortality.

J Gen Intern Med 35(7):2084-93

DOI: $10.1007 / \mathrm{s} 11606-020-05634-3$

(C) Society of General Internal Medicine 2020

\section{INTRODUCTION}

Overactive bladder $(\mathrm{OAB})$ can be defined as a constellation of urinary symptoms typically characterized by urgency, with or without urge urinary incontinence, and accompanied by frequency and nocturia ${ }^{1}$. Antimuscarinic medications, the firstline pharmacotherapy for $\mathrm{OAB}$, block the muscarinic receptors and inhibit involuntary detrusor contractions, thereby reducing urgency ${ }^{2,3}$. Although antimuscarinic agents are effective in bladder control, there are differences in the safety and tolerability profiles of individual agents, mainly driven by the differences in receptor selectivity subtypes and capability to cross the blood-brain barrier ${ }^{4-6}$. Antimuscarinics such as oxybutynin, tolterodine, trospium, and fesoterodine are nonselective as they have affinity for all muscarinic receptors (M1-M5), while others such as darifenacin and solifenacin are selective due to their high affinity for $\mathrm{M} 2 / \mathrm{M} 3$ receptors that are responsible for bladder contraction.

Although selective and non-selective agents have comparable efficacy, muscarinic receptor selectivity could offer advantages over non-selective agents with respect to adverse effects $^{4,7-12}$. On the basis of available evidence, antimuscarinic agents with selectivity for $\mathrm{M} 3$ over $\mathrm{M} 1$ and $\mathrm{M} 2$ receptors, limited CNS penetration, or both may offer a favorable balance of safety and efficacy in treating $\mathrm{OAB}$ together with reduced adverse cognitive effects in the older population ${ }^{13}$. Medications with strong anticholinergic properties such as antimuscarinics have been associated with cognitive and other adverse effects including risk of mortality in diverse samples of older adults ${ }^{14-25}$. Two recent studies have 
evaluated the risk of cardiovascular events and overall mortality with antimuscarinics and provided conflicting findings $^{26,27}$. Arana et al. found increased risk of cardiovascular events and mortality among adults using oxybutynin compared to tolterodine ${ }^{26}$. However, Margulis et al. found no differential risk of cardiovascular events and overall mortality among adults using antimuscarinic agents for $\mathrm{OAB}^{27}$.

Dementia and $\mathrm{OAB}$ are the most common comorbid conditions affecting older patients. The multifactorial disease pathology of dementia leads to a fourfold increase in urinary incontinence and $\mathrm{OAB}$, and urinary problems affect up to $90 \%$ of patients with dementia ${ }^{28,29}$. Although antimuscarinics are effective for $\mathrm{OAB}$, highly anticholinergic medications including antimuscarinics are considered inappropriate for patients with dementia ${ }^{30}$. Anticholinergic medication use in patients with dementia is of particular concern, because patients with dementia suffer from progressive cognitive decline due to damage to the cholinergic nervous system, and therefore, they are more susceptible to the central anticholinergic adverse effects of antimuscarinics ${ }^{31-33}$. However, no studies evaluated comparative safety of antimuscarinics in older adults with dementia. Therefore, the objective of this study is to examine the comparative risk of all-cause mortality among older adults with dementia and $\mathrm{OAB}$ using non-selective versus selective antimuscarinics. We hypothesized that non-selective antimuscarinics would lead to a higher risk of mortality than selective antimuscarinic agents in patients with dementia and $\mathrm{OAB}$.

\section{METHODS}

\section{Data Source}

The study used multiyear Medicare claims data from 2013 to 2015 including parts A, B, and D claim data files involving $100 \%$ of the national cohort of older patients with dementia and OAB. Medicare data files are available as Research Identifiable Files from the CMS upon request ${ }^{34,35}$. Medicare Standard Analytical Files (SAF) are available on yearly basis from the CMS. These data files are restricted to claims submitted by fee-for-service (FFS) enrollees. This study used the MedPAR File, Outpatient SAF, Carrier SAF, Master Beneficiary Summary File (MBSF), and Prescription Drug Event (PDE) files. The MBSF segment includes beneficiary enrollment information $(\mathrm{A} / \mathrm{B} / \mathrm{C} / \mathrm{D})$, in addition to demographic information. The PDE files include events from all beneficiaries participating in the part $\mathrm{D}$ program.

\section{Study Design}

A retrospective cohort design (Fig. 1) matched on propensity scores was used to examine the comparative risk of all-cause mortality in older patients with dementia and $\mathrm{OAB}$ using nonselective versus selective antimuscarinic medications. The entire study duration was from $01 / 01 / 2013$ to $12 / 31 / 2015$. Index use of antimuscarinic medications for the older patients with dementia and $\mathrm{OAB}$ was defined as the first prescription of antimuscarinic medication in the patient identification period (01/01/2014-12/31/2015) after 12 months of washout period (01/01/2013-12/31/2013) from the study start date. This ensured that (i) the study included only incident antimuscarinic users to reduce survivor bias among prevalent users and (ii) each patient had a minimum 12 months of baseline period to accurately identify any pre-existing comorbidities/ comedications that could influence treatment selection for OAB.

The index antimuscarinic use was classified as nonselective or selective antimuscarinic medication. Consistent with previous research ${ }^{23}$, these antimuscarinic users were followed up to a maximum of 6 months ( 180 days) from their index date to examine the risk of all-cause mortality with the use of non-selective versus selective antimuscarinic medications. Exclusion criteria also included use of antimuscarinic medications before dementia diagnosis and gaps in continuous enrollment.

\section{Exposures and Outcome Definitions}

Antimuscarinic medication exposure was identified via $\mathrm{Na}-$ tional Drug Codes (NDC) and generic names (Supplementary Table S1) using PDE files. The selective antimuscarinic cohort included new users of solifenacin or darifenacin. The nonselective antimuscarinic cohort included new users of oxybutynin, fesoterodine, tolterodine, or trospium. The primary outcome of this study was the time to all-cause mortality during the follow-up duration of maximum 6 months (180 days) after initiation of either non-selective or selective drug class (index date). The MBSF part A/B/C/D summary file was used to identify mortality events for all beneficiaries. Patients were followed until they died or were censored if they switched to the other antimuscarinic medication class from the index antimuscarinic medication class, or discontinued their antimuscarinic medication (gap of $\geq 7$ days between the last date of medication use and the end of follow-up period), or had a gap of $\geq 7$ days between two consecutive refills, or reached the end of their follow-up period or end of study period (December 31, 2015), whichever happened earlier.

\section{Cohort Matching}

Propensity score matching was used to minimize the differences in baseline characteristics between the two exposure groups such that they differed only based on antimuscarinic treatment assignment ${ }^{36,37}$. More than 50 covariates were included in the propensity score calculation based on previously published literature, expert opinions of clinicians and geriatricians, and their association with treatment and outcome 20, 22, 23, 26, 27 These included clinical characteristics such as comorbidities and comedications and socio-demographics such as age and gender. Comorbidities were captured using Elixhauser's index during the 12 months before the index 
Total Study Period from Jan 1, 2013 to Dec 31, 2015

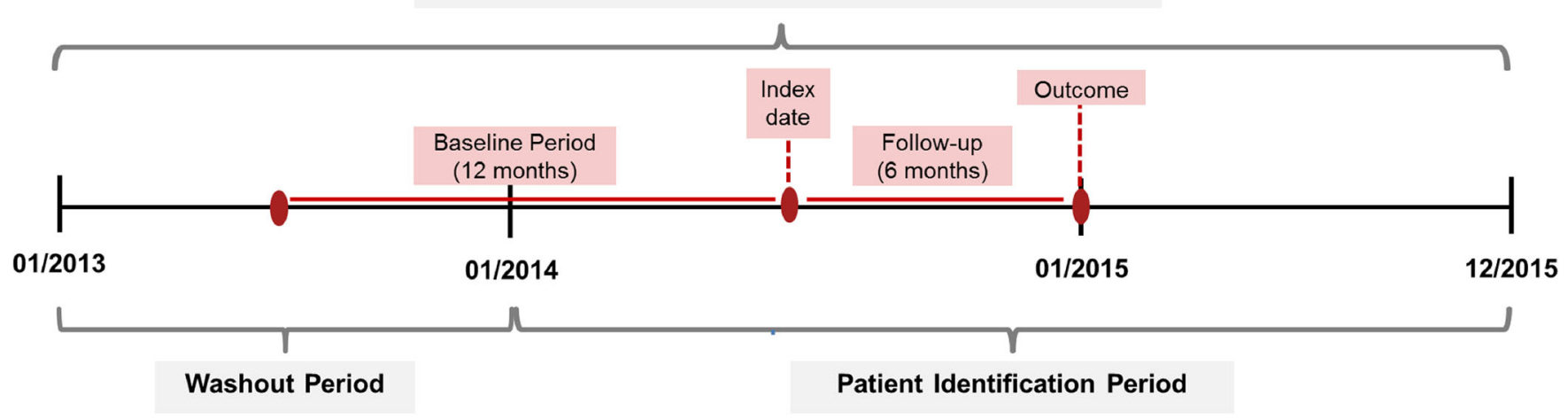

Figure 1 New antimuscarinic use study design.

date $^{38}$. In addition, the Anticholinergic Drug Scale (ADS) was used to account for the baseline anticholinergic load ${ }^{39}$. A logistic regression model was developed using all baseline covariates to predict exposure to selective antimuscarinics versus non-selective agents. Patients taking non-selective antimuscarinics were then matched on this predicted probability of exposure using the GREEDY $5 \rightarrow 1$ matching technique. This matching technique, in general, reduces matched-pair bias caused by incomplete and inexact matching ${ }^{40}$.

\section{Statistical Analysis}

An a priori total sample size of 1340 was estimated to yield $80 \%$ power to demonstrate a hazard ratio of 1.46 based on our previous work involving anticholinergics ${ }^{23}$. The covariate balance before and after matching was checked using bivariate analyses and standardized differences. Kaplan-Meier survival plots were created to depict the crude (unadjusted) relationship between non-selective versus selective antimuscarinic use and time to all-cause mortality. The proportionality hazard assumption, evaluated via the Schoenfeld residuals test, was supported by a non-significant relationship between residuals and time. The stratified Cox proportional-hazards model was conducted to evaluate the risk of all-cause mortality from nonselective antimuscarinic use (selective antimuscarinics as reference), within 180 days after initiating antimuscarinic treatment, with censoring. SAS 9.4 (SAS Institute, Cary, NC) was used for all analyses.

Several sensitivity analyses were conducted to evaluate the robustness of the study findings. The first sensitivity analysis evaluated the mortality risk among users of the most frequently reported individual non-selective versus selective antimuscarinic medication in the matched cohort. In addition, the risk of mortality was evaluated in the matched cohort within a maximum follow-up duration of 3 months (90 days). Since propensity score matching can limit generalizability, Cox proportional-hazards regression with stabilized inverse probability of treatment weighting (IPTW) was implemented on the entire unmatched sample ${ }^{41}$. Another sensitivity analysis was conducted to evaluate the mortality risk in the unmatched incident antimuscarinic user cohort using propensity score as a covariate. Finally, an unmeasured confounder analysis was conducted, whereby the strength of an unmeasured confounder was calculated based on the mortality risk estimates found in the study ${ }^{42}$. All analyses were conducted using an a priori $\alpha$ level of 0.05 .

The study was approved by the University of Houston Institutional Review Board Committee for the Protection of Human Subjects under the exempt category.

\section{RESULTS}

Figure 2 shows the process of identification of older adults with dementia and $\mathrm{OAB}$ who were new users of antimuscarinic medications. Overall, there were 3,383,603 Medicare beneficiaries diagnosed with dementia between 2013 and 2015. Over one million $(1,049,897)$ of these older adults with dementia had $\mathrm{OAB}$ for overall prevalence of $31.03 \%$. Among OAB patients, 287,612 (27.01\%) were prevalent antimuscarinics users during the study period; 79,397 (7.56\%) were incident antimuscarinic users in 2014-2015. After continuous eligibility criteria, the incident users included 21,848 (10.34\%) incident antimuscarinic users after dementia diagnosis.

Among the incident antimuscarinic users, 16,955 (77.6\%) were non-selective antimuscarinic users and 4893 (22.4\%) were selective antimuscarinic users; these antimuscarinic users were used for calculation of propensity scores. The most frequently reported non-selective antimuscarinic medications were oxybutynin $(12,295,72.5 \%)$ and tolterodine $(2868$, $16.9 \%$ ); among selective antimuscarinic agents, solifenacin $(4669,95.4 \%)$ was most frequently prescribed followed by darifenacin $(224,4.6 \%)$. Table 1 reports the differences in baseline characteristics between the two groups before and after matching. After matching, both the groups $(N=4862$ each) were similar in terms of distribution of the baseline 


\section{$4,701,621$}

All Medicare beneficiaries from Jan 1, 2013 to Dec 31, 2015

\section{$4,257,849$}

Aged 65 years and above as of study start date (Jan 1, 2013)

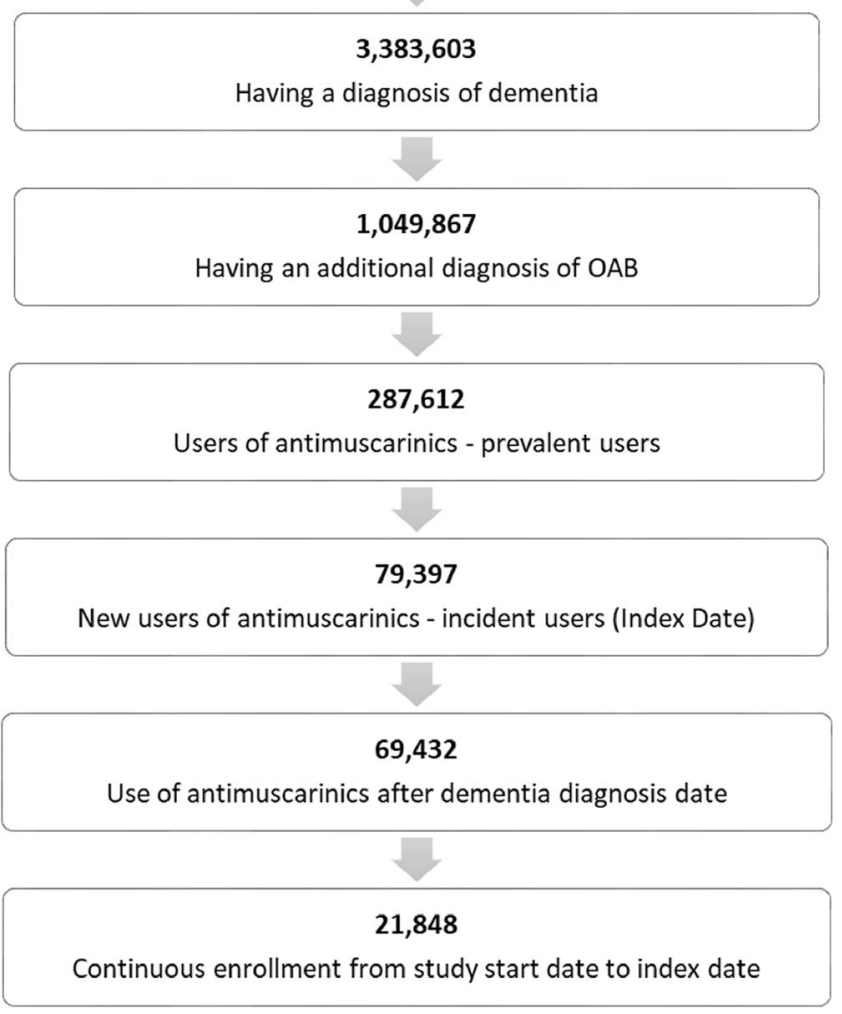

Figure 2 Cohort development flowchart.

characteristics and comparable in terms of distribution of the baseline characteristics. (Fig. 3).

\section{Risk of All-Cause Mortality}

The mortality rate was $2.3 \%$ (396) for non-selective and $1.6 \%$ (80) for selective antimuscarinic users in the total cohort $(p$ value $<0.01$ ) before matching. The rate of mortality was $2.6 \%$ (126) for non-selective and $1.6 \%$ (78) for selective antimuscarinic users in the matched cohort $(p$ value $<0.01)$. This translates to absolute risk reduction of $1 \%$ and number needed to harm of 100. Kaplan-Meier survival curves also revealed significant association between antimuscarinic use and risk of all-cause mortality ( $p$ value $<0.01$ ) (Fig. 4).

Table 2 presents results from the stratified Cox proportional-hazards model for the risk of all-cause mortality between non-selective and selective antimuscarinic users in the matched cohort. The Cox model found 50\% higher risk of 180-day mortality with non-selective antimuscarinics as compared to selective ones (hazard ratio (HR) 1.50; $95 \%$ confidence interval (CI) 1.04-2.16).

\section{Sensitivity Analysis}

Similar findings were observed with multiple sensitivity analyses (Table 2). A significantly increased hazard ratio was found (HR, 1.68; 95\% CI, 1.14-2.48) for the risk of allcause mortality between non-selective and selective antimuscarinic users in the matched cohort during the reduced followup period (90 days). Higher risk of all-cause mortality was also observed among older adults with dementia and $\mathrm{OAB}$ who initiated treatment with non-selective oxybutynin (HR 1.58; 95\% CI 1.06-2.34) as compared to selective solifenacin.

In the unmatched cohort, an increased mortality risk was observed when propensity score was used as a covariate (HR, 1.33; 95\% CI, 1.04-1.69). These findings remained consistent when propensity score weighting approach was used in the unmatched sample (IPTW HR, 1.43; 95\% CI, 1.13-1.74). Finally, the strength of unmeasured confounder was 2.36; this shows that the observed HR of 1.50 could be explained away by an unmeasured confounder that was associated with both the antimuscarinic use and mortality by a HR as high as 2.36fold each, above and beyond the measured confounders.

\section{DISCUSSION}

The study evaluated the risk of mortality in a national cohort of older adults with dementia using antimuscarinics for $\mathrm{OAB}$ and found that non-selective antimuscarinics were associated with a $50 \%$ increase in risk of all-cause mortality compared to selective antimuscarinics. The study findings remained consistent in multiple sensitivity analyses with $68 \%$ increased mortality risk within a 90-day follow-up and a 58\% increased risk of mortality with oxybutynin versus solifenacin use. In the unmatched cohort, a $43 \%$ increased risk of mortality was observed for the non-selective antimuscarinic group in propensity score weighing approach and a $33 \%$ increased mortality risk was observed when propensity score was used as a covariate.

This is the first study to the authors' knowledge that evaluated the comparative risk of mortality for non-selective versus selective antimuscarinics among older patients with dementia and OAB. Previous research by Arana et al. based on UK's Clinical Practice Research Datalink involving patients 18 years or older reported a $26 \%$ increased risk of overall mortality among oxybutynin users and 32\% reduced risk among solifenacin users when compared to tolterodine users ${ }^{26}$. However, Marguilis et al. did not report any difference in cardiovascular, stroke, acute myocardial infarction, or major acute cardiac event-related mortality risk with use of individual antimuscarinic agents compared to tolterodine ${ }^{27}$. The higher mortality risk reported in the current study cannot be directly compared with the findings of Arana et al. due to differences in study sample, namely older adults with dementia and the comparator used, selective agents ${ }^{26}$.

Our study findings confirm the study hypothesis that nonselective antimuscarinics are associated with a higher risk of 


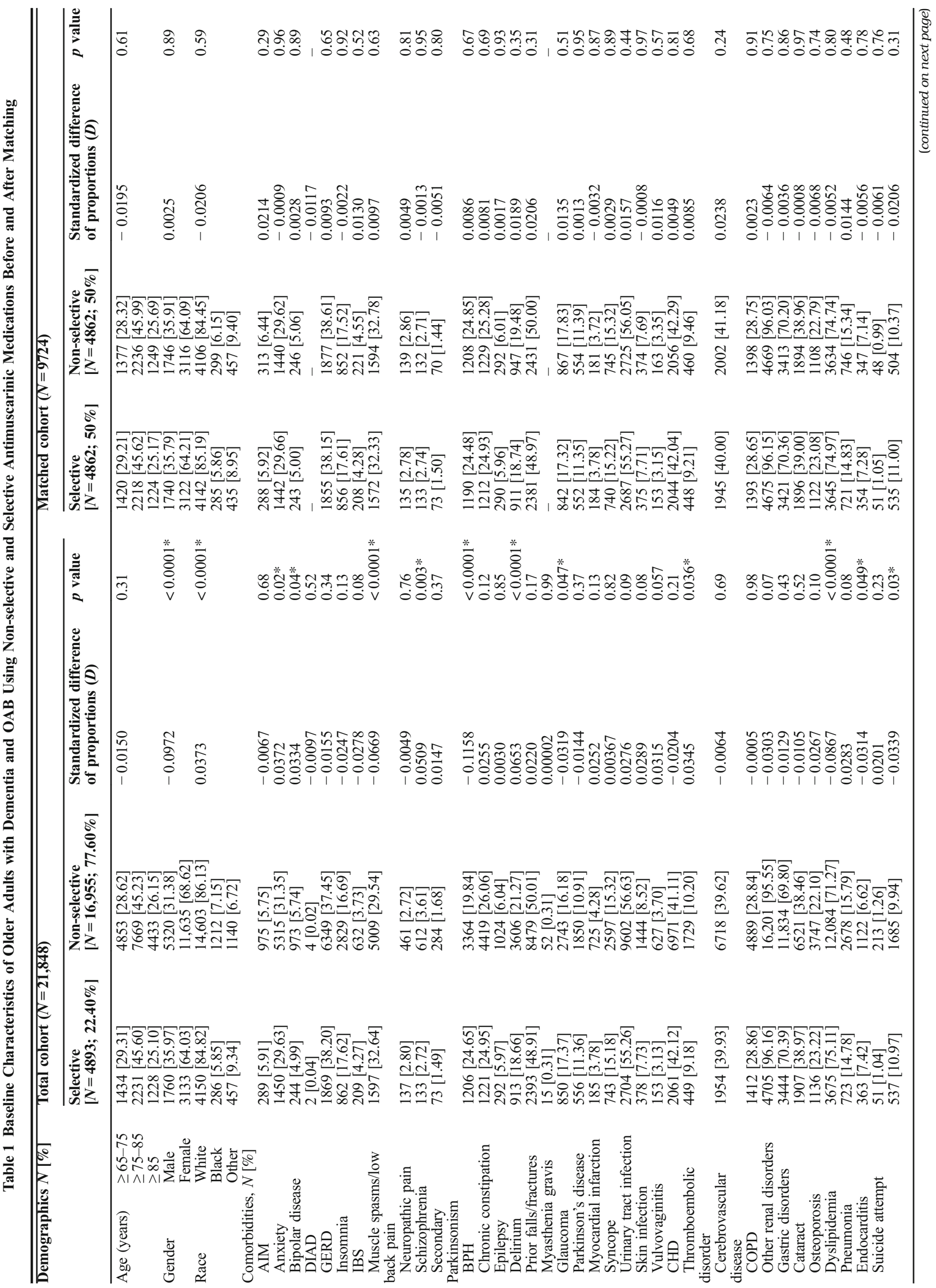




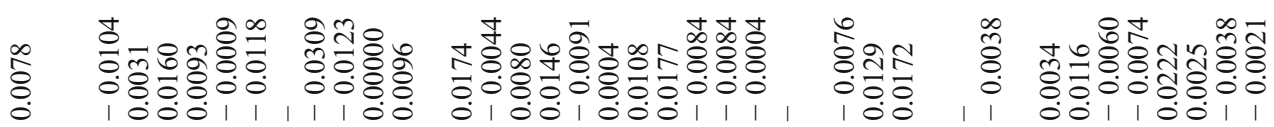

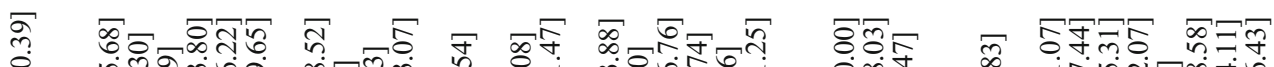

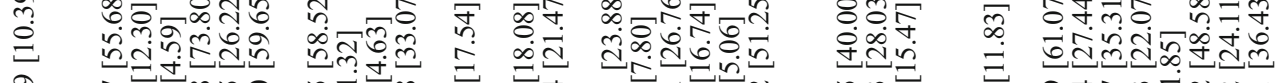

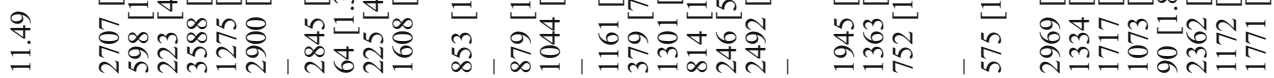

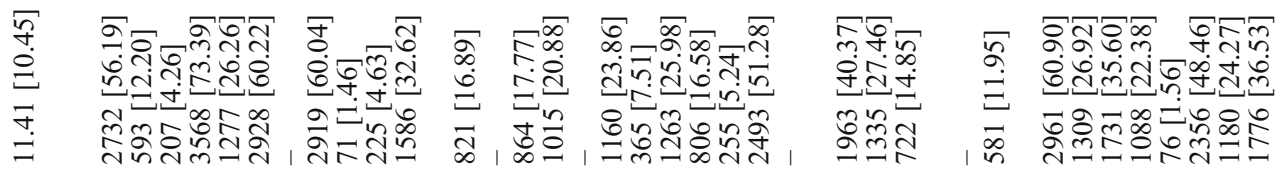

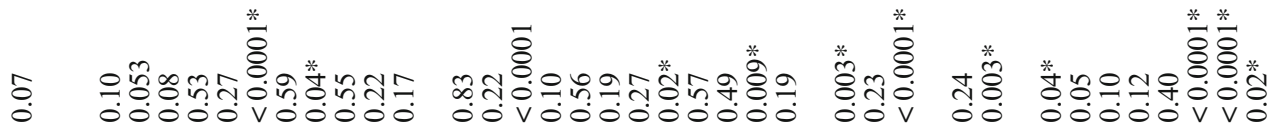

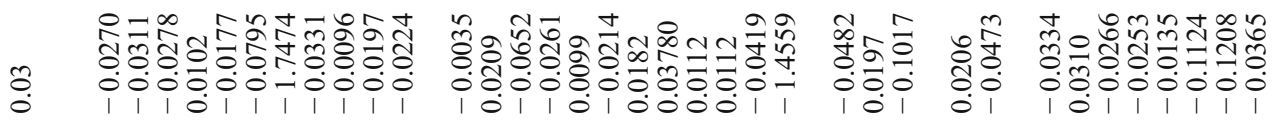

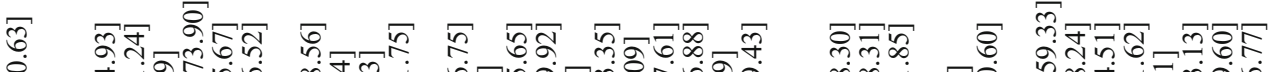

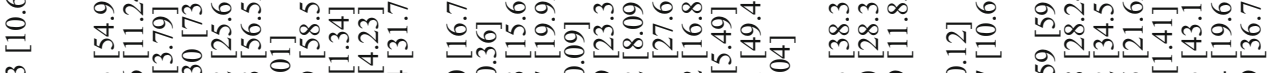

至

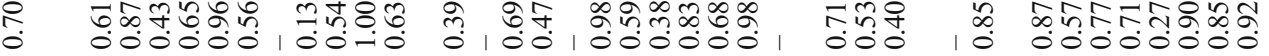

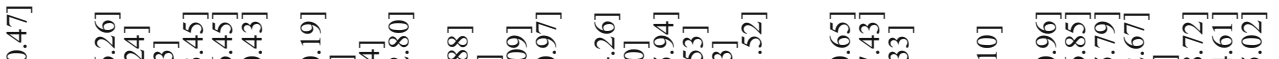

药

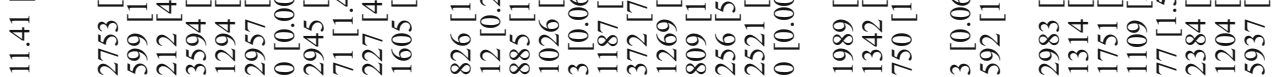


a

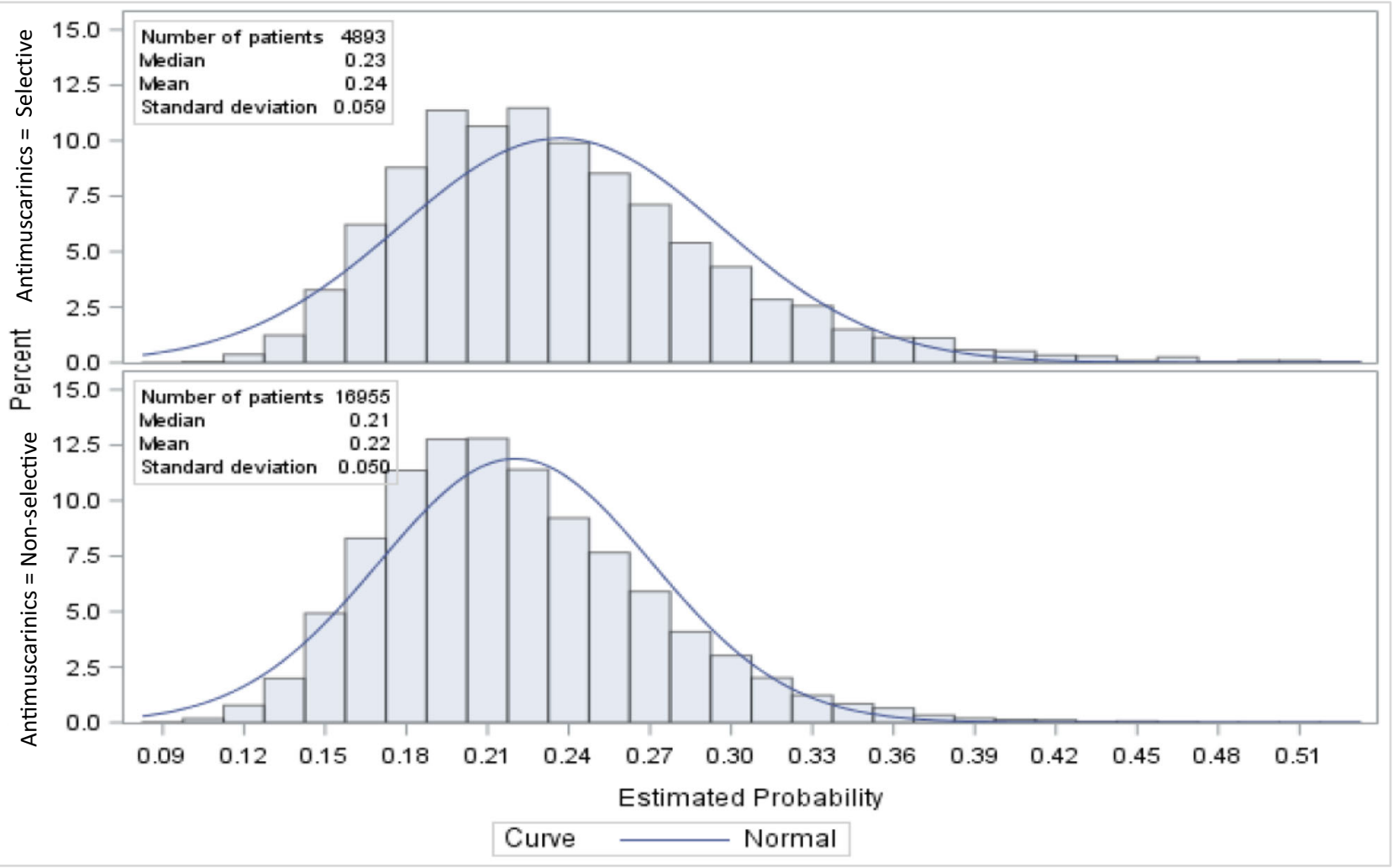

b

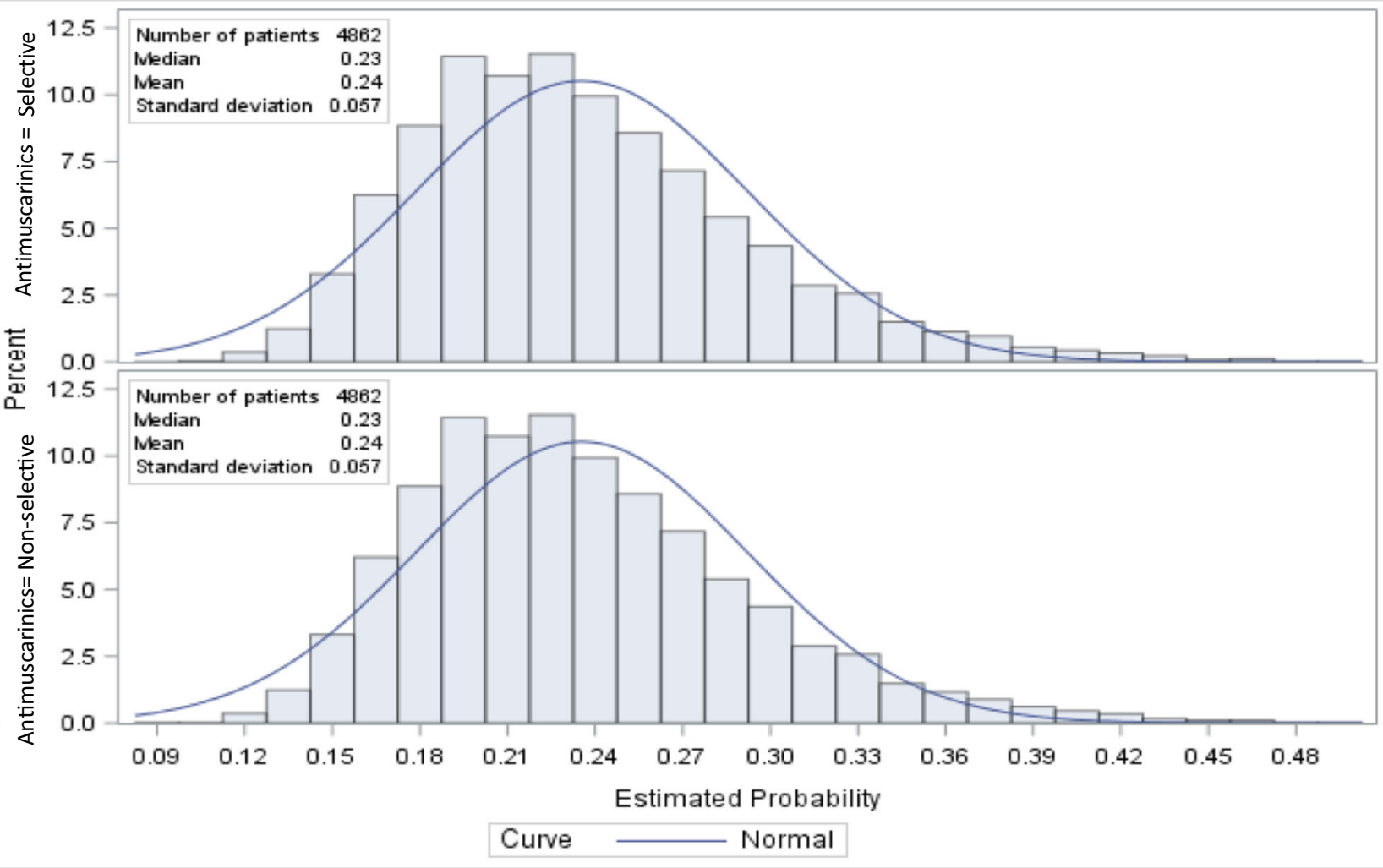

Figure 3 Distribution of propensity scores among non-selective and selective antimuscarinic medication users before (a) and after (b) matching. 


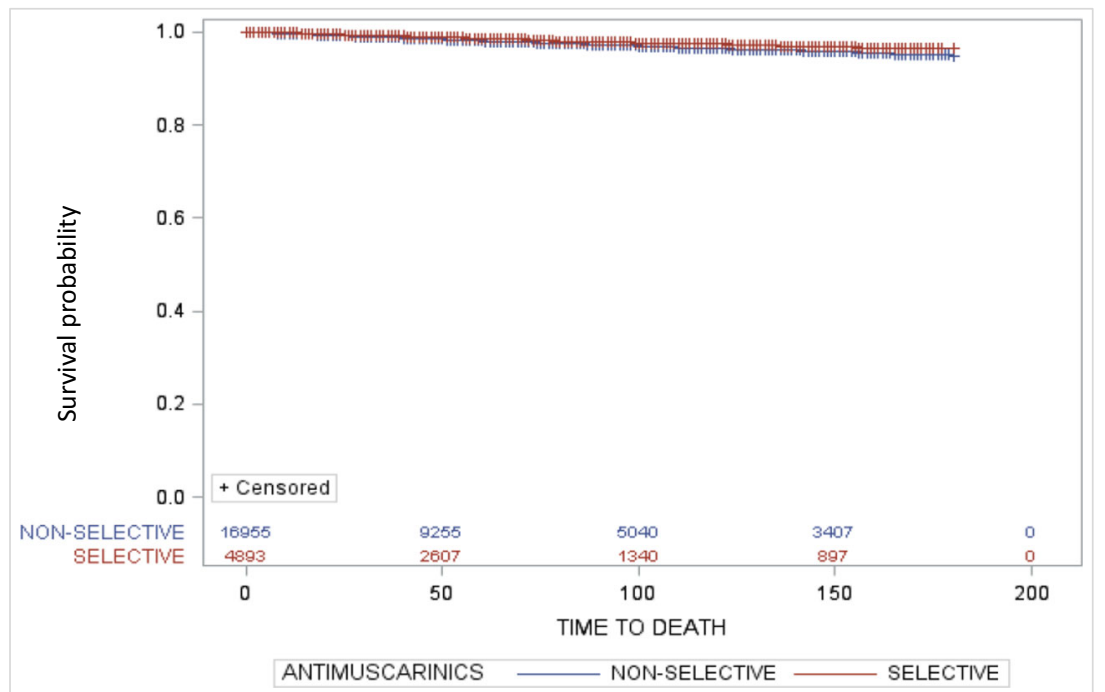

Figure 4 Kaplan-Meier plot of crude association between non-selective and selective antimuscarinic medication users and risk of all-cause mortality. Log-rank test: $p$ value $=\mathbf{0 . 0 0 8}$.

mortality than selective antimuscarinic agents in patients with dementia and OAB. The adverse effects of non-selective antimuscarinics are a consequence of their binding to different receptor subtypes that have minimal or no involvement in bladder detrusor contractions, such as M1 and M4 muscarinic receptors ${ }^{7}$. This leads to central and peripheral adverse events including heart rate, secretions, pneumonia, sedation, visual disturbances, and others. The effect of M1/M4 is particularly problematic in dementia as inhibition of the M1 receptors leads to adverse cognitive effects ${ }^{7}$. Oxybutynin, the first non-selective drug approved for OAB in 1975, has a slightly higher affinity for $\mathrm{M} 1$ and $\mathrm{M} 3$ receptors than for $\mathrm{M} 2$ receptors 43. Additionally, the pro-arrhythmic and pro-ischemic effects of non-selective agents can attribute to their increased cardiovascular risk 44,45 .

Solifenacin and darifenacin, on the other hand, are selective muscarinic M3 receptor antagonists; the relatively high selectivity of darifenacin for the M3 receptors as compared to the M1 receptors minimizes cognitive adverse effects 46,47 . Therefore, the study findings strongly suggest that selective antimuscarinics can offer a favorable safety profile over nonselective antimuscarinics for the management of $\mathrm{OAB}$ in patients with dementia. It should be noted that the current study did not evaluate the specific reasons for mortality. Therefore, more research is needed to understand the role of specific mechanisms contributing to increased mortality risk due to non-selective antimuscarinics in older patients with dementia.

The high use of non-selective antimuscarinic medications, especially oxybutynin, further raises concerns. There was a $58 \%$ increased risk of all-cause mortality among oxybutynin users compared to those using solifenacin. Given the relatively higher utilization of oxybutynin $(n=12,295 ; 56 \%)$ observed in the overall population, there is a significant need to reduce

Table 2 Stratified Cox Proportional-Hazards Model for Risk of All-Cause Mortality Due to Non-selective vs Selective Antimuscarinic Medication Use in Older Adults with Dementia and OAB

\begin{tabular}{|c|c|c|c|}
\hline Exposure & Hazard ratio (HR) & 95\% confidence interval $[95 \% \mathrm{CI}]$ & $\overline{p \text { value }}$ \\
\hline \multicolumn{4}{|l|}{ Main analysis } \\
\hline Non-selective antimuscarinic $[N=4862]$ & 1.50 & $1.04-2.16$ & \multirow[t]{2}{*}{$0.03 *$} \\
\hline Selective antimuscarinic $[N=4862]$ & 1.00 & Reference & \\
\hline \multicolumn{4}{|l|}{ Sensitivity analysis } \\
\hline Mortality risk with the most frequently reported antimus & in each category (ox & vs solifenacin) & \\
\hline Non-selective antimuscarinic - oxybutynin $[N=4624]$ & 1.58 & $1.06-2.34$ & \multirow{2}{*}{$0.02 *$} \\
\hline Selective antimuscarinic - solifenacin $[N=4624]$ & 1.00 & Reference & \\
\hline \multicolumn{4}{|l|}{ Mortality risk within maximum follow-up of 90 days } \\
\hline Non-selective antimuscarinic $[N=1201]$ & 1.68 & $1.14-2.48$ & \multirow[t]{2}{*}{$0.008 *$} \\
\hline Selective antimuscarinic $[N=1201]$ & 1.00 & Reference & \\
\hline \multicolumn{4}{|l|}{ Mortality risk in unmatched sample using PS as covariate } \\
\hline Non-selective antimuscarinic $[N=16,955]$ & 1.33 & $1.04-1.69$ & \multirow[t]{2}{*}{$0.02 *$} \\
\hline Selective antimuscarinic $[N=4893]$ & 1.00 & Reference & \\
\hline \multicolumn{4}{|l|}{ Inverse probability of treatment weight analysis } \\
\hline Non-selective antimuscarinic $[N=16,955]$ & 1.43 & $1.13-1.74$ & \multirow[t]{2}{*}{$0.003 *$} \\
\hline Selective antimuscarinic $[N=4893]$ & 1.00 & Reference & \\
\hline
\end{tabular}

*Statistical significance at $p$ value $<0.05$ 
oxybutynin use in older adults with dementia. The higher use of oxybutynin compared to other antimuscarinics could be due to its preferred tier status that may lead physicians to prefer this drug compared to other antimuscarinics ${ }^{48}$. Also, antimuscarinic treatments other than oxybutynin may not be affordable to some patients based on insurance coverage. This poses a considerable challenge in the management of $\mathrm{OAB}$ in older adults with dementia. Given the high mortality risk and adverse cognitive safety profile of oxybutynin, the better safety profiles with selective antimuscarinic agents observed in the current study provide support for expanding patient access to include the selective antimuscaranics as preferred agents, especially for older adults with dementia. Careful screening of patients for cognitive deficits prior to any non-selective antimuscarinic treatment can also be one of the ways to improve antimuscarinic prescribing.

This study had several strengths including the study design and analytical approach used. A new-user retrospective cohort design based on propensity-matched approach provided an efficient method of assessing the causal relationship between the antimuscarinic exposure and risks of mortality. Although randomization of exposure did not take place, this pseudorandomization technique of propensity score matching strengthened our study findings. The study used relevant covariates in the propensity score model including baseline anticholinergic exposure and Elixhauser's index, a widely used risk adjustment tool proven to be statistically superior for predicting various outcomes including mortality. Additionally, the main findings did not change when propensity score weighting approach was used to increase the generalizability.

\section{Limitations}

The study findings should be interpreted in the light of potential limitations. Due to the use of claims data, actual use of medication could not be ascertained. However, by allowing only a 7-day gap in medication use, a conservative definition of continued medication persistence was applied which may have excluded patients who stopped the medication. The diseases, exposures, and outcome measurements were based on medical claims and could be subject to coding issues. Variables included for the propensity-matched cohort were limited to those available in the data source; hence, residual confounding due to unmeasured covariates such as physician preferences and other clinical measures might have affected the study findings. Although the study controlled for patients with high baseline anticholinergic load, the dosage of these medications was not controlled. Our sensitivity analysis revealed that an unmeasured confounder must be strongly associated with a HR of 2.36 with the treatment and the outcome to explain away the association. The use of prescription data did not include use of over-the-counter drugs and prescription drugs in inpatient settings. The study is limited to Medicare beneficiaries in community settings; therefore, future studies are needed to assess the safety of antimuscarinics in diverse settings.

\section{CONCLUSIONS}

The current study found that non-selective antimuscarinic use was associated with 50\% increased risk of mortality compared to selective agents in older adults with dementia and OAB. The findings remained consistent across several sensitivity analyses. The study findings suggest that physicians should prefer selective antimuscarinics over non-selective agents to manage $\mathrm{OAB}$ in older adults with dementia. Expanding patient access for selective agents can also help to improve quality of care in dementia. Given the increased risk of allcause mortality observed in this study, future research should focus on cause-specific mortality and other drug-related morbidity.

Acknowledgments: The authors would like to thank Dr. Satabdi Chatterjee for the editorial and statistical assistance in preparation of the manuscript.

Corresponding Author: Rajender R. Aparasu, PhD, FAPhA; Department of Pharmaceutical Health Outcomes and Policy, College of Pharmacy University of Houston, Houston, TX, USA (e-mail: rraparasu@uh.edu).

Primary Funding Source This study was supported by a grant from the National Institutes of Aging (NIA) (Grant R15AG056997; Principal Investigator: Rajender R. Aparasu). The funding agency had no role in study design, data collection and analysis, decision to publish, or preparation of the manuscript.

\section{Compliance with Ethical Standards:}

The study was approved by the University of Houston Institutional Review Board Committee for the Protection of Human Subjects under the exempt category.

Conflict of Interest: Dr. Aparasu has received research funding from Astellas Inc., Incyte Corp., and Novartis Inc. for projects unrelated to the current work. No other disclosures are reported.

\section{REFERENCES}

1. Haylen BT, de Ridder D, Freeman RM, et al. An International Urogynecological Association (IUGA)/International Continence Society (ICS) joint report on the terminology for female pelvic floor dysfunction. Neurourol Urodyn. 2010; 29(1): 4-20.

2. Haab F. Chapter 1: the conditions of neurogenic Detrusor overactivity and overactive bladder. Neurourol Urodyn. 2014; 33 Suppl 3:S2-5.

3. Chess-Williams R. Muscarinic receptors of the urinary bladder: detrusor, urothelial and prejunctional. Auton Autacoid Pharmacol, 2002; 22(3): 33-45.

4. Chapple C, Khullar V, Gabriel Z, Dooley JA. The effects of antimuscarinic treatments in overactive bladder: a systematic review and metaanalysis. Eur Urol. 2005; 48(1): 5-26.

5. Kessler TM, Bachmann LM, Minder C, et al. Adverse event assessment of antimuscarinics for treating overactive bladder: a network metaanalytic approach. PLoS One. 2011; 6(2): e16718.

6. Andersson K-E, Campeau L, Olshansky B . Cardiac effects of muscarinic receptor antagonists used for voiding dysfunction. $\mathrm{Br} \mathrm{J}$ Clin Pharmacol 2011; 72:186-196

7. Glavind $\mathbf{K}$, Chancellor $\mathbf{M}$. Antimuscarinics for the treatment of overactive bladder: understanding the role of muscarinic subtype selectivity. Int Urogynecol J. 2011; 22(8): 907-17. 
8. Peretto I, Petrillo P, Imbimbo BP. Medicinal chemistry and therapeutic potential of muscarinic M3 antagonists. Med Res Rev. 2009; 29 (6):867902.

9. Abrams P, Andersson KE. Muscarinic receptor antagonists for overactive bladder. BJU Int. 2007; 100(5): 987-1006.

10. Appell RA. Pharmacotherapy for overactive bladder: an evidence-based approach to selecting an antimuscarinic agent. Drugs. 2006; 66(10): 1361-70.

11. Michel MC, Barendrecht MM, Oelke M. Anticholinergics for overactive bladder: does subtype selectivity play a role? Urologe A. 2006; 45(7): 8269.

12. Abrams P, Andersson KE, Buccafusco JJ, et al. Muscarinic receptors: their distribution and function in body systems, and the implications for treating overactive bladder. Br J Pharmacol. 2006; 148(5): 565-78.

13. Kay GG, Abou-Donia MB, Messer WS Jr, et al. Antimuscarinic drugs for overactive bladder and their potential effects on cognitive function in older patients. J Am Geriatr Soc. 2005; 53(12): 2195-201

14. Campbell N, Boustani M, Limbil T, et al. The cognitive impact of anticholinergics: a clinical review. Clin Interv Aging. 2009; 4: 225-33.

15. Chatterjee S, Carnahan RM, Chen H, et al. Anticholinergic medication use and risk of pneumonia in elderly adults: a nested case-control study. J Am Geriatr Soc. 2016; 64(2): 394-400.

16. Richardson $\mathbf{K}$, Bennett $\mathbf{K}$, Maidment ID, et al. Use of medications with anticholinergic activity and self-reported injurious falls in older community-dwelling adults. J Am Geriatr Soc. 2015; 63(8):1561-9.

17. Dauphinot V, Faure R, Omrani S, et al. Exposure to anticholinergic and sedative drugs, risk of falls, and mortality: an elderly inpatient, multicenter cohort. J Clin Psychopharmacol. 2014; 34(5): 565-70.

18. Aizenberg $\mathbf{D}$, Sigler M, Weizman A, et al. Anticholinergic burden and the risk of falls among elderly psychiatric inpatients: a 4-year case-control study. Int Psychogeriatr. 2002; 14(3): 307-10.

19. Chatterjee S, Bali V, Carnahan RM, et al. Anticholinergic medication use and risk of fracture in elderly adults with depression. J Am Geriatr Soc. 2016; 64(7):1492-7.

20. Vetrano DL, La Carpia D, Grande G, et al. Anticholinergic medication burden and 5-year risk of hospitalization and death in nursing home elderly residents with coronary artery disease. J Am Med Dir Assoc. 2016; 17(11): 1056-59.

21. Nishtala PS, Narayan SW, Wang T, et al. Associations of drug burden index with falls, general practitioner visits, and mortality in older people. Pharmacoepidemiol Drug Saf. 2014; 23(7):753-8.

22. Ruxton K, Woodman RJ, Mangoni AA. Drugs with anticholinergic effects and cognitive impairment, falls and all-cause mortality in older adults: a systematic review and meta-analysis. $\mathrm{Br} \mathrm{J}$ Clin Pharmacol. 2015; 80(2): 209-20.

23. Chatterjee S, Bali V, Carnahan RM, et al. Risk of mortality associated with anticholinergic use in elderly nursing home residents with depression. Drugs Aging. 2017; 34(9): 691-700.

24. Coupland CAC, Hill T, Dening T, et al. Anticholinergic drug exposure and the risk of dementia: a nested case-control study. JAMA Intern Med 2019; 179(8): 1084-1093.

25. Moga DC, Abner EL, Wu $\mathbf{Q}$, et al. Bladder antimuscarinics and cognitive decline in elderly patients. Alzheimers Dement (N Y). 2017; 3(1): 139-148.

26. Arana A, Margulis AV, McQuay LJ, et al. Variation in cardiovascular risk related to individual antimuscarinic drugs used to treat overactive bladder: a UK cohort study. Pharmacotherapy. 2018; 38(6): 628-37.

27. Margulis AV, Hallas J, Pottegård A, et al. Comparison of cardiovascular events among treatments for overactive bladder: a Danish nationwide cohort study. Eur J Clin Pharmacol. 2018; 74(2):193-9.

28. Hellstrom $\mathbf{L}$, Ekelund $\mathbf{P}$, Milsom I, et al. The influence of dementia on the prevalence of urinary and fecal incontinence in 85-year-old men and women. Arch Gerontol Geriatr. 1994; 19:11-20

29. Skelly J, Flint A. Urinary incontinence associated with dementia. J Am Geriatr Soc. 1995; 43 (3): 286-94.
30. American Geriatrics Society 2015 Beers Criteria Update Expert Panel American Geriatrics Society 2015 Updated Beers Criteria for potentially inappropriate medication use in older adults. J Am Geriatr Soc.; 63(11): 2227-46.

31. Kemper RF, Steiner V, Hicks B, et al. Anticholinergic medications: use among older adults with memory problems. J Gerontol Nurs. 2007; 33(1): 21-31.

32. Rudolph JL, Salow MJ, Angelini MC, et al. The anticholinergic risk scale and anticholinergic adverse effects in older persons. Arch Intern Med. 2008; 168(5): 508-13.

33. Lechevallier-Michel N, Molimard M, Dartigues JF, et al. Drugs with anticholinergic properties and cognitive performance in the elderly: results from the PAQUID Study. Br J Clin Pharmacol. 2005; 59(2): 14351.

34. Research Data Assistance Center (ResDAC): Available CMS Data Available at https://www.resdac.org/file-availability (Accessed May 2019)

35. CMS Chronic Conditions Data Warehouse (CCW) Data Dictionaries Available at https://ccwdata.org/web/guest/data-dictionaries (May 2019)

36. Baser O. Too much ado about propensity score models? Comparing methods of propensity score matching. Value Health. 2006; 9(6): 377-85.

37. Brookhart MA, Schneeweiss S, Rothman KJ, et al. Variable selection for propensity score models. Am J Epidemiol. 2006; 163(12): 1149-56.

38. Elixhauser A, Steiner C, Harris DR, et al. Comorbidity measures for use with administrative data. Med Care. 1998; 8-27.

39. Carnahan RM, Lund BC, Perry PJ, et al. The Anticholinergic Drug Scale as a measure of drug-related anticholinergic burden: associations with serum anticholinergic activity. J Clin Pharmacol. 2006; 46 (12): 1481-6.

40. Parsons LS. Reducing Bias in a Propensity Score Matched-Pair Sample Using Greedy Matching Techniques. In Proceedings of the Twenty-Sixth Annual SAS Users Group International Conference. SAS Institute Inc.; 2001.

41. Austin PC, Stuart EA. Moving towards best practice when using inverse probability of treatment weighting (IPTW) using the propensity score to estimate causal treatment effects in observational studies. Stat Med. 2015;34:3661-3679.

42. VanderWeele TJ, Ding $\mathbf{P}$. Sensitivity analysis in observational research introducing the E-value. Ann Intern Med. 2017;167(4):268-74.

43. Norhona-Blob L, Kachur, JF. Enantiomers of oxybutynin: in vitro pharmacological characterization at M1, M2 and M3 muscarinic receptors and in vivo effects on urinary bladder contraction, mydriasis and salivary secretion in guinea pigs. J Pharmacol Exp Ther 1991; 256(2): 562-7.

44. Cetinel B, Onal B, Gultekin MH, et al. Which antimuscarinic agents used in the treatment of overactive bladder increase heart rate? A prospective randomized clinical trial. Int Urol Nephrol. 2019;51(3):417424

45. Rosa GM, Bauckneht M, Scala C, et al. Cardiovascular effects of antimuscarinic agents in overactive bladder. Expert Opin Drug Saf. 2013;12:815-27.

46. Napier C, Gupta P. Darifenacin is selective for the human recombinant M3 receptor subtype. Neurourol Urodyn. 2002; 21(4): A445.

47. Wesnes K, Lipton $\mathbf{R}$, Kolodner $\mathbf{K}$, et al. Darifenacin, an M3 selective receptor antagonist for the treatment of overactive bladder, does not affect cognitive function in elderly volunteers. Eur Urol. 2004; 3(2):131.

48. Vouri SM, Schootman M, Strope SA, et al. Differential prescribing of antimuscarinic agents in older adults with cognitive impairment. Drugs Aging. 2018; 35(4): 321-31.

Publisher's Note Springer Nature remains neutral with regard to jurisdictional claims in published maps and institutional affiliations. 